\title{
Underwater Acoustic Signatures of Recreational Swimmers, Divers, Surfers and Kayakers
}

\author{
Christine Erbe $^{1}$ (D) Miles Parsons ${ }^{1} \cdot$ Alec J. Duncan $^{1} \cdot$ Kim Allen $^{1}$
}

Received: 18 March 2016 / Accepted: 20 June 2016 / Published online: 8 July 2016

(C) Australian Acoustical Society 2016

\begin{abstract}
Non-motorised, recreational water activities were recorded underwater in the controlled setting of a public swimming pool during the off-season. Individuals, one at a time, swam freestyle and breaststroke, snorkelled, scuba-dived, kicked a boogie board and a surfboard, kayaked, and simply jumped into the water. Underwater video and still images were recorded at the same time to interpret the sounds recorded. Most of the sound was due to bubbles generated underwater. Activities involving fins (flippers) were the loudest (boogie boarding and snorkelling), followed by freestyle swimming, surfboard paddling, and kayaking. Breaststroke generated the fewest bubbles and was the quietest. All activities produced bubbles, hence noise, at a characteristic temporal pattern. Scuba-diving exhibited two distinct noise spectra related to inhalation and exhalation. Received levels ranged from 110 to $131 \mathrm{~dB}$ re $1 \mu \mathrm{Pa}(10-16,000 \mathrm{~Hz})$ for all of the activities at the closest point of approach $(1 \mathrm{~m})$. The results might have applicability to the monitoring of pools for security reasons, to performance assessments of swimmers, and to studies of the distances at which humans may be detectible by marine animals in the sea.
\end{abstract}

Keywords Sound signature $\cdot$ Swimming performance $\cdot$ Kayaking $\cdot$ Diving $\cdot$ Man-made sound $\cdot$ Underwater sound

\section{Introduction}

Underwater sound generated by humans has been studied for several decades, as part of environmental impact assessments of industrial operations, e.g. onshore construction, shipping, offshore petroleum exploration, and naval manoeuvres, or as part of defence and naval warfare research and development (e.g. [1,2]). Underwater sound from non-industrial, and non-motorised activities, like recreational swimming, is sensibly expected to be much weaker and possibly negligible and therefore has received less attention. This article presents the results of a study to determine the underwater sound signatures of recreational water activities. Three hydrophones were installed in a swimming pool and people were recorded swimming in different styles, snorkelling, scuba-diving, kicking a boogie board and a surfboard, kayaking, and simply

Christine Erbe

c.erbe@curtin.edu.au

1 Centre for Marine Science and Technology, Curtin University, Kent Street, Bentley, WA 6102, Australia jumping into the water. We present spectrograms and spectra of the corresponding underwater sounds, explain the physical origin of the various sounds, most of which are related to bubbles underwater, and discuss potential applications of this data, e.g. the monitoring of people in the water, including performance assessments of swimmers.

\section{Methods}

Underwater acoustic recordings were made on 19 May 2015 in a public freshwater swimming pool in Perth, Western Australia. The pool was $50 \mathrm{~m}$ long and $25 \mathrm{~m}$ wide. Three omnidirectional hydrophones (Teledyne Reson TC 4033, bandwidth $1 \mathrm{~Hz}-140 \mathrm{kHz}$, receiving sensitivity $-202 \mathrm{~dB}$ re $1 \mathrm{~V} / \mu \mathrm{Pa}$ ) were deployed in a row, along one of the $50-\mathrm{m}$ long lanes, at $4.5 \mathrm{~m}$ range from the long wall, and at 10, 14, and $20 \mathrm{~m}$ from the short wall, respectively. All three channels were simultaneously recorded on a Sound Devices 744T portable audio recorder (24 bit, $18 \mathrm{~dB}$ internal gain on each channel, sampling frequency $32 \mathrm{kHz}$ ). The external pream- 
Table 1 Summary of activities that were recorded

\begin{tabular}{lll}
\hline Style & $\begin{array}{l}\text { Number of different } \\
\text { people recorded }\end{array}$ & $\begin{array}{l}\text { Total number of } \\
\text { times recorded }\end{array}$ \\
\hline Freestyle swimming & 5 & 45 \\
Breaststroke swimming & 5 & 48 \\
Snorkelling with fins & 3 & 27 \\
Kicking a boogie board with fins & 1 & 18 \\
Lying on a surfboard, kicking & 3 & 27 \\
$\quad$ without fins and paddling with & & \\
$\quad$ alternating arms & 1 & 9 \\
Kneeling on a surfboard and & & \\
$\quad$ paddling synchronously with & & 18 \\
both arms & 2 & 12 \\
Kayaking & 1 & 30 \\
Fruba-diving & 3 & \\
\hline
\end{tabular}

plifiers were models Etec B1501 (40 dB gain) on Channels 1 and 2, and Teledyne Reson EC6061/VP1000 (32 dB gain) on Channel 3. The hydrophones were installed in $1.8 \mathrm{~m}$ of water, $80 \mathrm{~cm}$ above the bottom of the pool, mounted on laboratory stands and clamps that included sponge padding on feet and clamps to limit noise. Each channel was calibrated with input white noise prior to the recordings, showing a flat frequency response $( \pm 1 \mathrm{~dB})$ between $2 \mathrm{~Hz}$ and $16 \mathrm{kHz}$.

Five recreational swimmers participated in this study, swimming one at a time, along the 50-m lane, over the three hydrophones. The styles recorded were (1) freestyle, (2) breaststroke, (3) snorkelling with fins (flippers), (4) lying on a boogie (body) board kicking with fins, (5) lying on a surfboard kicking without fins and paddling with alternating arms, (6) kneeling on a surfboard paddling synchronously with both arms, (7) paddling a sit-on-top kayak, and (8) scuba-diving. All of the activities (1)-(7) happened at the water surface, $1 \mathrm{~m}$ above the hydrophones. Activity 8, scubadiving, however, was recorded while the diver swam at $1 \mathrm{~m}$ depth below the surface, i.e. at the height of the hydrophones, but along a lane at $1 \mathrm{~m}$ horizontal distance from the row of hydrophones. Finally, free jumps and head-dives were also recorded, at a horizontal distance $>3 \mathrm{~m}$ from the hydrophone on Channel 1.

Table 1 lists the different activities recorded, the number of people who performed each activity, and the total number of samples collected, in other words, the total number of times each activity was recorded (over all people and hydrophones).

During the recordings, all pumps and machinery, including the filtration system of the pool, were switched off. Ambient noise was recorded for $5 \mathrm{~min}$ at the beginning. Aerial and underwater still images and video were also taken.

All acoustic recordings were stored as .wav files, and analysed with custom-written software in MATLAB R2015b (The MathWorks Inc., Natick, MA). Power spectral den- sity spectrograms were generated by converting the recorded voltage time series to pressure, then using the Welch (Fourier transform based) method of power spectrum estimation [3] with Hamming windows of $50 \%$ overlap. The closest point of approach (CPA) was determined from each spectrogram, as the time of maximum broadband power. A 1-s window was selected around the CPA for each pass over each hydrophone, and the mean power spectrum, as well as the 95th percentile were computed for each activity.

\section{Results}

Figure 1 shows aerial and underwater images of the various recreational activities recorded. Common to all images are bubbles, the dominant source of sound in all activities.

Figure 2 shows the 20-s spectrograms for seven of the recreational water activities. All of the sounds emitted were broadband, covering the full $16-\mathrm{kHz}$ bandwidth of recording. A distinct multipath (Lloyd's mirror) interference pattern was seen in the spectrogram for each of the activities, particularly those happening at the water surface. For sound sources in shallow water, the signal arrivals at a hydrophone via the direct, surface-reflected and bottom-reflected paths interfere constructively and destructively at different frequencies that vary with time as the sound source approaches and then moves away from the hydrophone. This is seen in the spectrogram as a downward/upward sloping pattern before/after the CPA. In Fig. 2, this Lloyd's mirror pattern is discernible at the lower frequencies in the spectrograms A, B and D. It is more obvious in Fig. 3, which zooms into the lower frequencies of the spectrogram for about 1 min of kicking a boogie board with fins.

The spectrograms in Fig. 2 exhibit different temporal patterns. Kicking a boogie board while wearing fins generated temporally continuous sound (Fig. 2A). The sound was due 

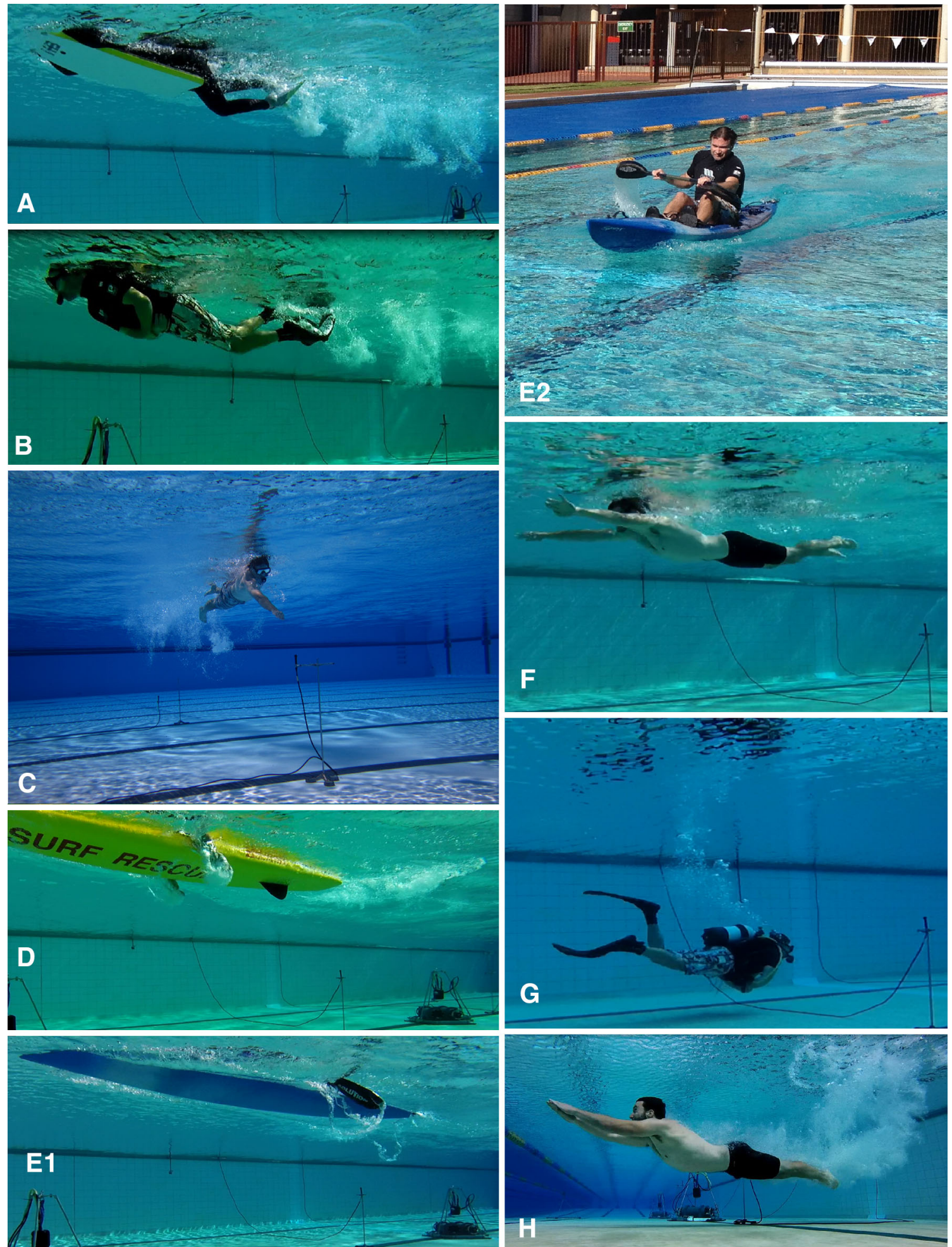

Fig. 1 Photographs of people A kicking a boogie board with fins, B snorkelling with fins, C freestyle swimming, D kneeling on a surfboard paddling with both arms simultaneously, E1 kayaking, E2 kayaking — note water running off the blade, F breaststroke swimming, $\mathbf{G}$ scuba-diving, and $\mathbf{H}$ free jumping 
Fig. 2 Spectrograms of seven different recreational water activities (NFFT $=4096$, Hamming window, 50\% overlap, fs $=32 \mathrm{kHz}$ ): A kicking a boogie board with fins, B snorkelling with fins, $\mathbf{C}$ freestyle swimming, D kneeling on a surfboard and paddling synchronously with both arms, $\mathbf{E}$ kayaking, $\mathbf{F}$ breaststroke swimming, G scuba-diving. All spectrograms show power spectral density on a colour scale of $45-125 \mathrm{~dB}$ re $1 \mu \mathrm{Pa}^{2} / \mathrm{Hz}$, in the frequency band $30 \mathrm{~Hz}-16$ $\mathrm{kHz}$, over a 20 -s duration, as the person performing the activity was moving past one of the hydrophones at a distance of $1 \mathrm{~m}$
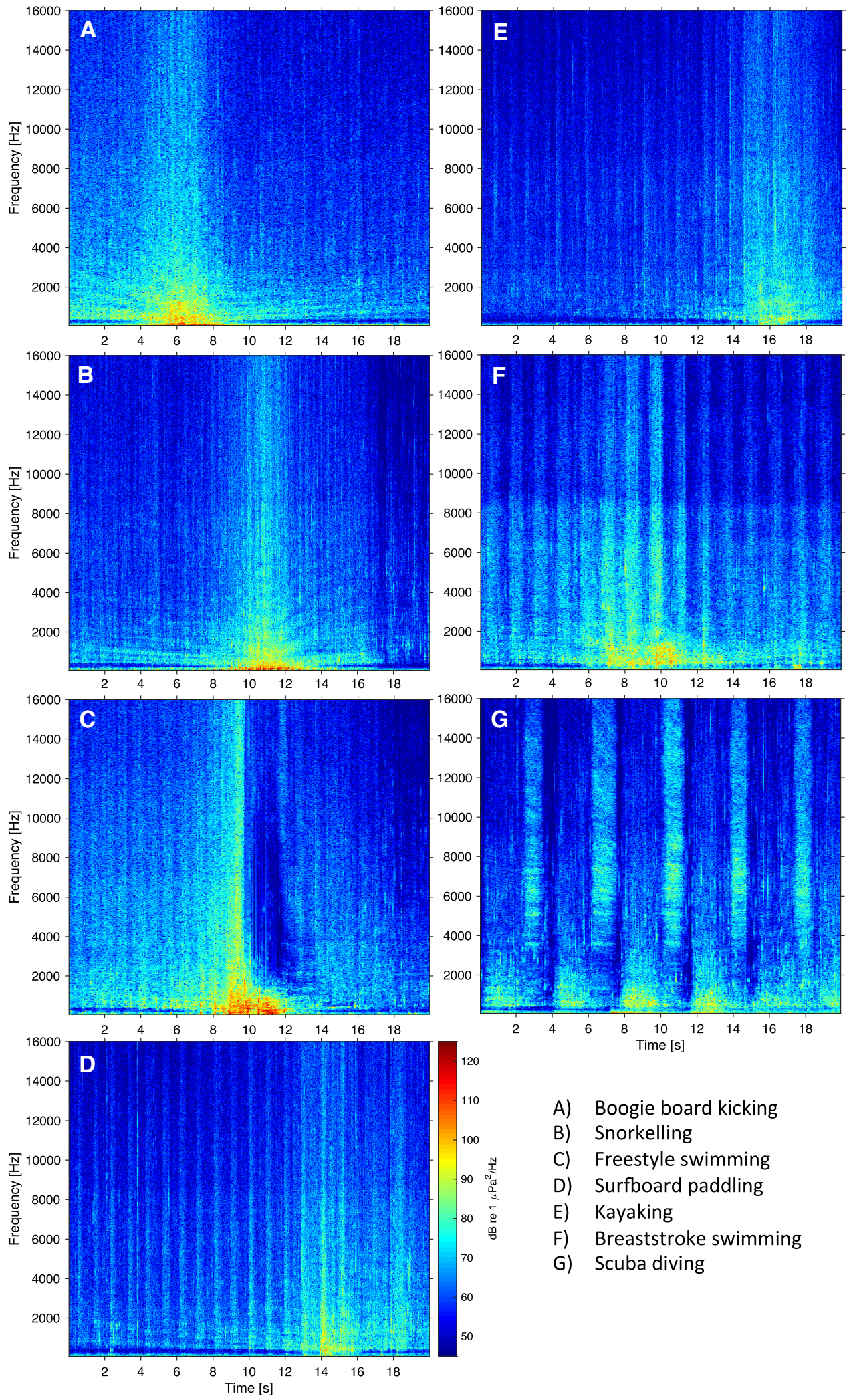
A) Boogie board kicking
B) Snorkelling
C) Freestyle swimming
D) Surfboard paddling
E) Kayaking
F) Breaststroke swimming
G) Scuba diving 
primarily to dense bubble clouds created by the strong and quick paddling action of the fins, and secondarily to bubbles in the wake of the boogie board (Fig. 1A).

Some of the people snorkelling with fins were kicking more slowly than the boogie boarder, and the sound of snorkelling of these swimmers consisted of strong periodic bursts at a repetition rate of $2 \mathrm{~Hz}$ (Fig. 2B). This corresponded to each leg kicking about once per second. The underwater bubble clouds from the last three kicks can clearly be seen in Fig. 1B.

All freestyle swimmers kicked their legs faster than other swimmers with fins, yielding a temporally continuous sound. One of the swimmers pushed the bubble cloud deep into the water, sometimes reaching the hydrophone at $1 \mathrm{~m}$ below the water surface, which led to low spectral values immediately after the CPA, as is seen in Fig. 2C from 10 to $12 \mathrm{~s}$ at $1-12$ $\mathrm{kHz}$.

Kneeling on a surfboard and paddling synchronously with both arms produced the strongly amplitude-modulated spectrogram in Fig. 2D. The arms went into the water about once per second. The bubbles pushed into the water by both hands can clearly be seen in Fig. 1D, in addition to the bubble wake of the surfboard.

Lying on a surfboard, kicking with both feet and rotating the arms alternately produced a very similar spectrogram to Fig. 2D, except that bubbles from arm action were generated twice as quickly, i.e. twice per second, with each arm entering the water once per second. Given that the surfboard was much longer than the boogie board, only the feet, rather than the full legs, were involved in kicking action, and this was much weaker hence quieter than that for freestyle, snorkelling or kicking on a boogie board.

Kayaking was quieter still (see Fig. 2E). There was some sound from bubbles at the paddle blades (see Fig. 1E1), bubbles in the wake, and bubbles generated by water periodically running off the opposite (in-air) blade of the paddle (Fig. 1E2).

Breaststroke produced almost no bubbles at the arms or legs. Bubbles were only generated during breathing, when the head pierced the water surface and when the swimmers exhaled underwater (Fig. 1F). The spectrogram was strongly amplitude-modulated, as a result of the breathing pattern (Fig. 2F).

The spectrogram of scuba-diving (Fig. 2G) showed a lowand a high-frequency component. Regions of high power from 3 to $16 \mathrm{kHz}$, peaking at $7 \mathrm{kHz}$, corresponded to airflow through the oxygen valve during inhalation and sounded like strained breathing. Regions of high power below $3 \mathrm{kHz}$ corresponded to bubbles produced during exhalation (Fig. 1G).

Figure 3 shows a spectrogram of about 1 min of kicking a boogie board while wearing fins, focussing on frequencies below $2 \mathrm{kHz}$. The Lloyd's mirror pattern is clearly visible. Further, there is a peak in the spectrum at around $80 \mathrm{~Hz}$.
Note that, after an initial rapid reduction in level around CPA, there is very little further reduction in the received level at frequencies around $80 \mathrm{~Hz}$ as the source moves further from the hydrophone.

Figure 4 presents mean spectra of the various activities as well as 95th percentiles. Overall, boogie board kicking and snorkelling produced the strongest sounds, due to the large volume of bubbles created by kicking with fins. Freestyle swimming followed, and, judging from underwater video and still images, it produced bubble clouds from both arms and legs, but these bubble clouds were less dense than those produced by kicking with fins. One freestyle swimmer produced more bubbles from his feet than the others, which is why the 95th percentile of the freestyle spectrum also shows the bubble cloud peak below $100 \mathrm{~Hz}$, which the freestyle mean does not show. Paddling with the arms, while kneeling or lying on a surfboard, and kayaking with a paddle produced weaker sounds. Small bubble volumes were generated in the wake of the board and kayak, and from the paddles. Breaststroke produced hardly any bubbles from the arms or legs, but only from the head during breathing, and was the weakest in the band 100-1000 Hz. Scuba-diving sound consisted of two parts: inhaling and exhaling. Inhaling produced sound above $3 \mathrm{kHz}$, as shown. Exhaling was stronger at lower frequencies.

Free jumping and diving off the side of the pool was recorded at distances $>3 \mathrm{~m}$. These were the strongest sounds, had a sudden onset (Fig. 5), and were accompanied by the largest bubble volumes (Fig. $1 \mathrm{H}$ ).

Figure 6 repeats the mean recorded spectra from five of the recreational activities of this study, and compares them with a sound recorded from dhufish [4], the median source spectrum of jetskis [5], underwater ambient sound off a beach (recorded by the authors), and wind-dependent, ambient noise at four sea states [6]. Jetskis are amongst the quieter motorised vessels in terms of their underwater noise, with boats and ships being noisier.

\section{Discussion}

This article presented some underwater sounds created by recreational water activities. The underwater sound recorded at frequencies above $200 \mathrm{~Hz}$ was mostly associated with the creation of bubbles in the water. Large and dense bubble clouds were generated by activities at the water surface that involved wearing fins (kicking a boogie board with fins and snorkelling with fins). Activities that involved less contact with the water surface (breaststroke swimming) or happened below the water surface (scuba diving) were quieter. The scuba diver also wore fins, but these did not penetrate the water surface, but rather were kicked at $1 \mathrm{~m}$ depth and produced no bubbles. 
Fig. 3 Underwater sound from kicking a boogie board with fins. The boogie boarder moved over the hydrophone once, then stopped, turned around and came back. The top panel shows the corresponding spectrogram zoomed into frequencies below $2 \mathrm{kHz}$ (NFFT = 8192, Hamming window, $50 \%$ overlap, fs $=32$ $\mathrm{kHz}$ ). The Lloyd's mirror effect is clearly visible as the V-shaped spectral pattern. The lower panel has the broadband frequency spectrum (power spectral density, PSD) computed as the mean over a 1.5 -s window around each of the two CPAs $(\mathrm{NFFT}=16000$, Hamming window, $50 \%$ overlap, $\mathrm{fs}=32 \mathrm{kHz})$
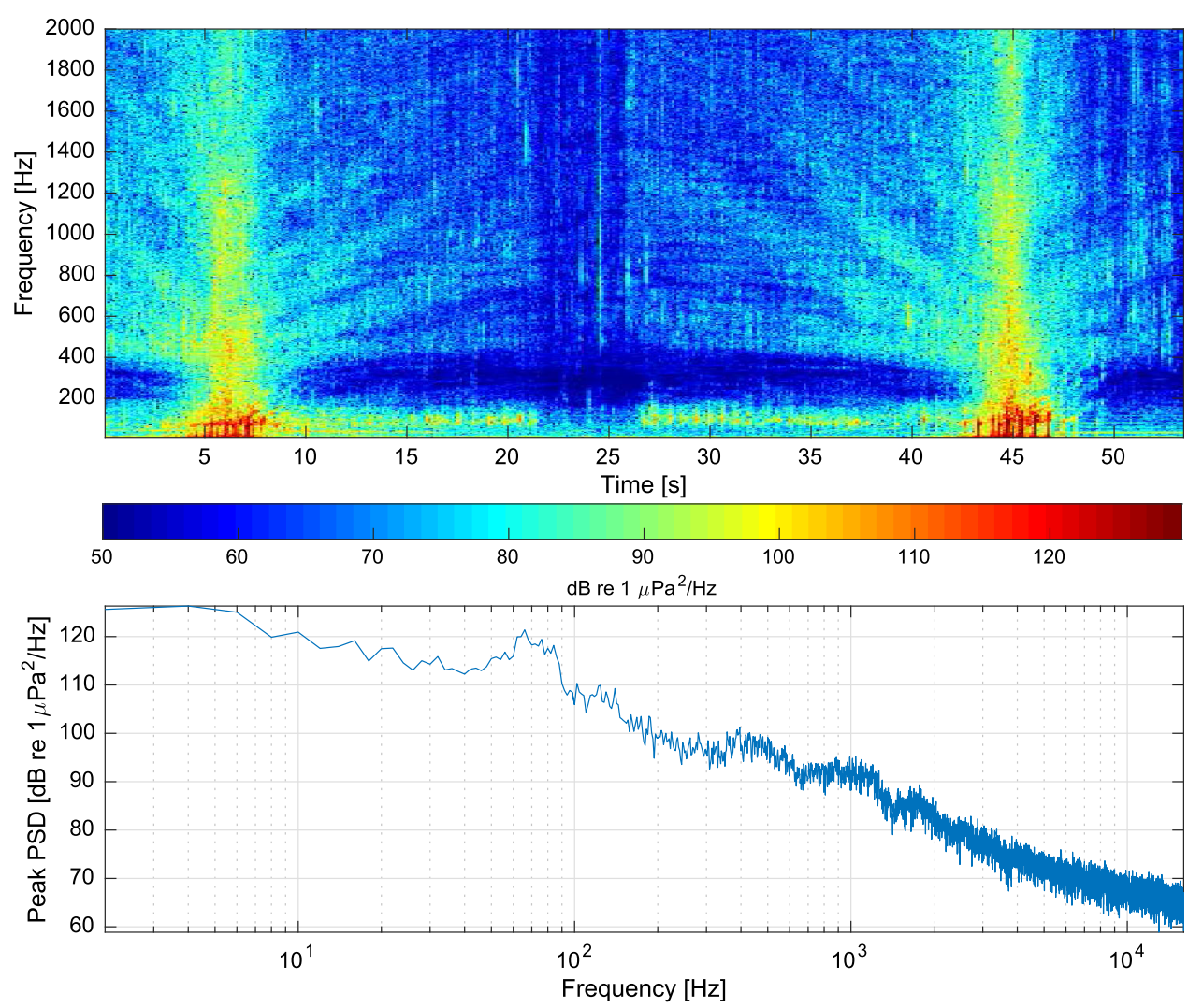
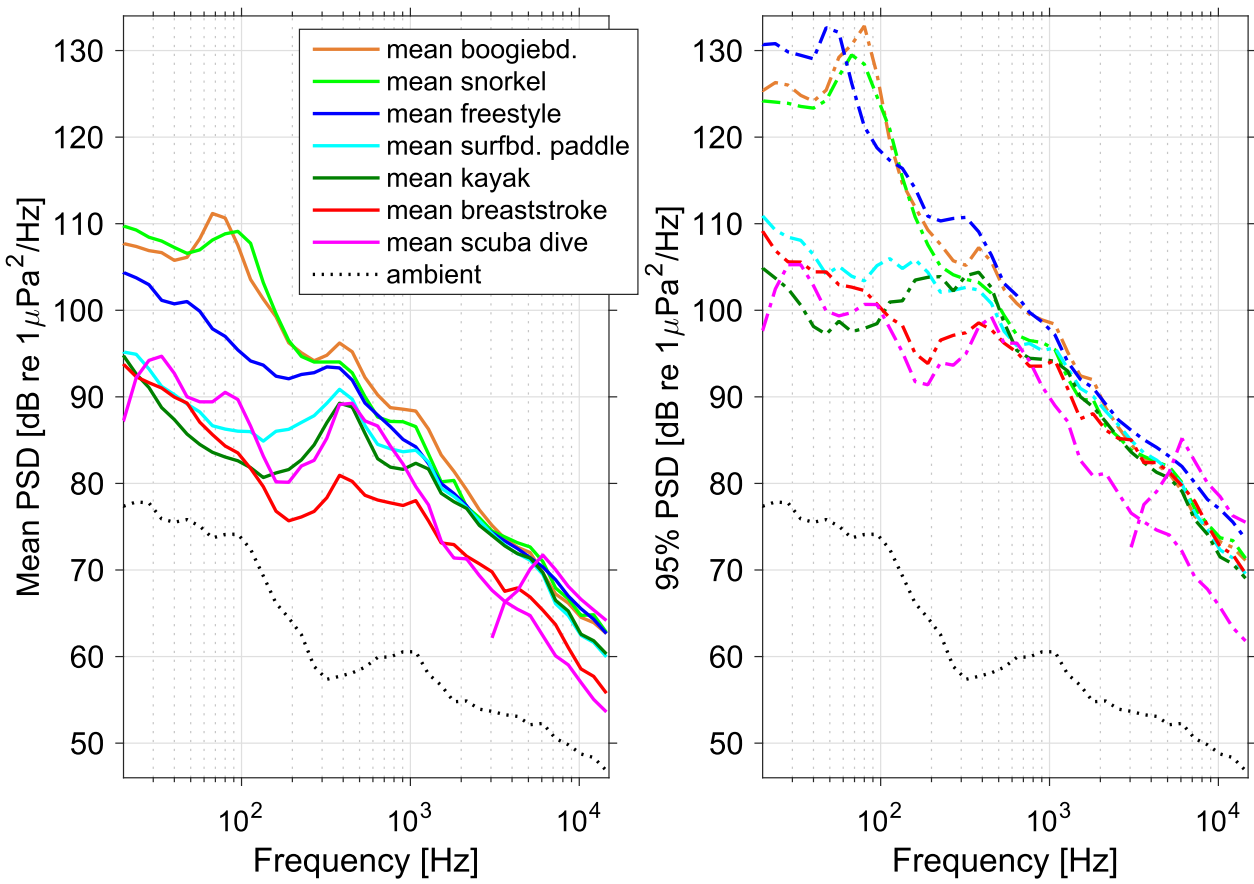

Fig. 4 Mean power spectral density of recreational water activities (left $)$ and 95 th percentiles $(r i g h t)$ over all passes within $1 \mathrm{~m}$ of the hydrophones. Spectra were computed over a 1-s window around CPA (NFFT $=8192$, Hamming window, $50 \%$ overlap, fs $=32 \mathrm{kHz}$ ). The coloured lines, as per the figure legend, give the performed activities. There are two spectra for scuba-diving: exhaling over the full bandwidth, and inhaling between 3 and $16 \mathrm{kHz}$ only as the inhaling spectrum was not discernible from ambient noise at lower frequencies. The mean ambient underwater noise spectrum in the pool when all machinery was switched off and no people were in the water is shown in both plots (dotted black) 

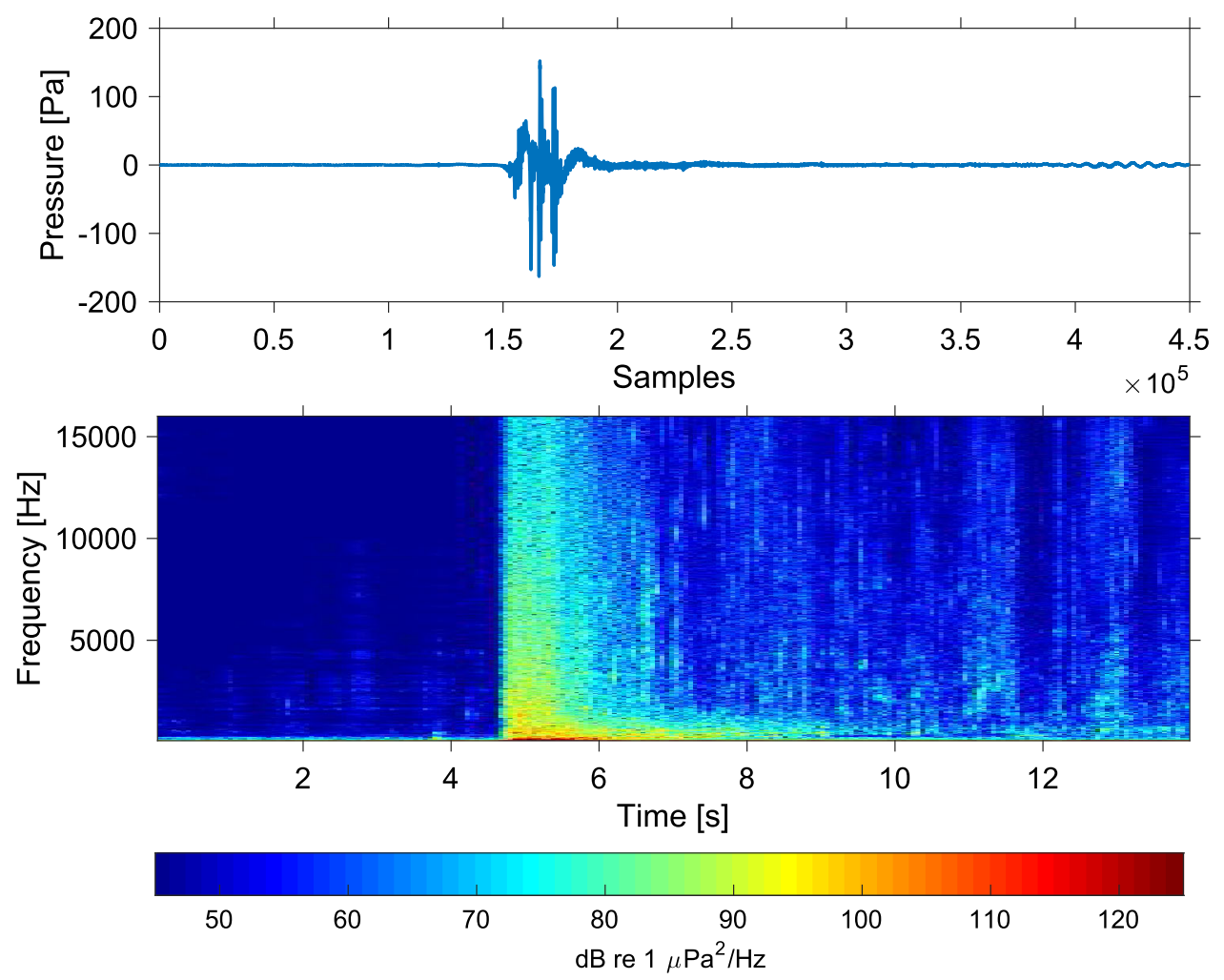

Fig. 5 Pressure time series and power spectral density spectrogram recorded from a free jump into the pool at $3 \mathrm{~m}$ from the hydrophone (NFFT $=$ 4096, Hamming window, $50 \%$ overlap, fs $=32 \mathrm{kHz}$ )

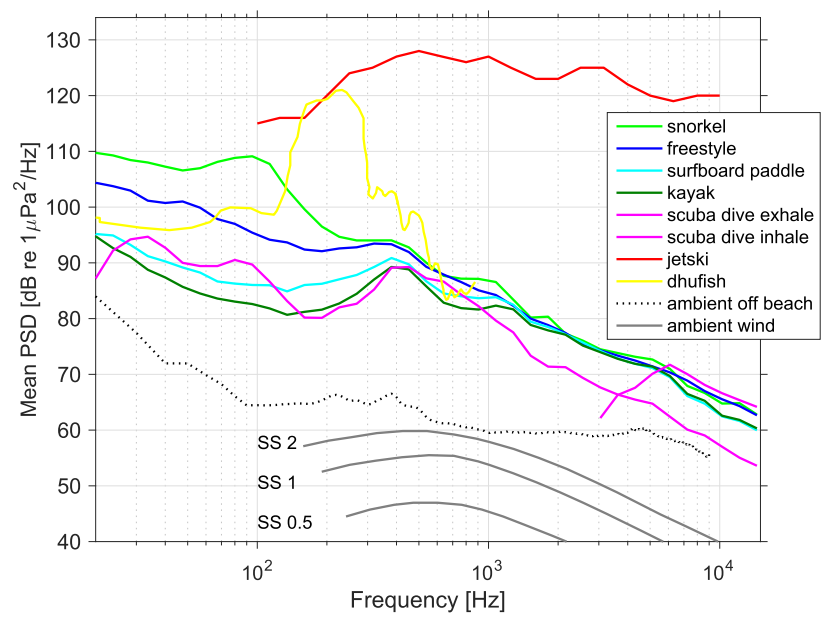

Fig. 6 Mean spectra of recreational water activities from this study, compared with the median source spectrum of jetskis [5], a source spectrum recorded from dhufish [4], ambient underwater noise off a beach, and ambient wind-dependent noise at sea states $0.5,1$, and 2 [6]. Ambient noise off a beach was recorded by us authors with a recorder on the seafloor in $7 \mathrm{~m}$ of water, $250 \mathrm{~m}$ off a local beach, Western Australia, behind the surf zone. Shown is the median spectrum over 73 days of recording from January to March 2015. The spectrum is dominated by the sound of waves breaking on the beach, in particular at low frequencies $(<100 \mathrm{~Hz})$. Small boats and jetskis dominate at mid frequencies $(200-800 \mathrm{~Hz})$, while snapping shrimp shape the spectrum at high frequencies $(3-8 \mathrm{kHz})$
An oscillating gas bubble underwater produces a travelling sound wave, and the sound of bubbles and bubble clouds has been reported in the literature (e.g. $[7,8])$. The typical spectrum drops at a rate of $-6 \mathrm{~dB}$ per frequency octave $(=-20 \mathrm{~dB}$ per frequency decade $)$ at frequencies greater than the resonance frequency. This slope is also seen in the spectra of the recreational water activities (Fig. 4) above 1 $\mathrm{kHz}$.

While oscillating bubbles create sound, they can also scatter and absorb sound (e.g. $[9,10])$. This interplay of bubble sound generation and attenuation was seen in some of the spectrograms, where a notch in the received spectrum appeared immediately after CPA (Fig. 2C). Similarly, several of the recorded passes were louder during the approach than departure as the bubble cloud behind the person attenuated some of the acoustic energy (e.g. Fig. 2A, B).

The mechanism for the production of the signals recorded on the hydrophones at frequencies below $200 \mathrm{~Hz}$ is less clear than at higher frequencies, and it is likely that the structure of the pool and/or the characteristics of the ground underneath it, were having an influence on the measured results at these low frequencies. In several cases (boogie boarding, snorkelling, and scuba-diving) there was a distinct peak in the spectrum at around $80 \mathrm{~Hz}$ (Fig. 3). These frequencies are too low for this noise to be explainable by the oscillation of individual bubbles, but there are several other possible mech- 
anisms that could account for it. These include the oscillation of each cloud of bubbles as a single entity, and the hydrodynamic pressure fluctuations caused by the changes in water flow over moving arms, legs, fins or paddles. An oscillating bubble cloud is an efficient radiator of sound, whereas hydrodynamic pressure fluctuations result in a pressure field that decays relatively quickly away from the source, with only a small component radiating into the far-field [8]. In this case, the CPA distance of approximately $1 \mathrm{~m}$ was similar to the length scale of the sources, so either or both of these mechanisms could be responsible for the energy in this frequency range.

Another observation from Fig. 3 was that, after an initial rapid reduction in level around CPA, there was very little further reduction in the received level at frequencies around $80 \mathrm{~Hz}$ as the source moved further from the hydrophone. This contrasted with levels at higher frequencies that showed a distinct reduction in level out to the maximum distance. This appears to be a consequence of the acoustic propagation conditions in the pool. At $80 \mathrm{~Hz}$ the acoustic wavelength in water is over $18 \mathrm{~m}$, or more than 10 times the water depth. Any acoustic propagation in the pool at these frequencies must therefore also involve vibration of the structure of the pool and/or the ground beneath the pool. It is therefore likely that low-frequency sound or pressure fluctuations associated with the activity were exciting such vibrations which were then coupling to the hydrophone either through the water or through the stand supporting the hydrophone.

Overall, the recorded spectrum is a superposition of the direct sound at the hydrophones and multiple reflections from the pool bottom and water surface, plus potential low-frequency vibration, all adding together. The spectrum recorded at CPA depends on the depth of the hydrophone. In open water, the spectrum might be different, depending on the depth and reflectivity of the seafloor. We therefore presented the spectra as received levels at $1 \mathrm{~m}$ below the water surface, rather than source levels, which could be applied to various environments.

Comparing the spectra recorded from the recreational water activities with a few other sounds underwater, dhufish (one of the quieter sound producing fish species) were more powerful at around $200 \mathrm{~Hz}$, and jetskis (amongst the quietest motorised vessels) were more powerful over the entire bandwidth reported. The spectra were, however, above those of wind-dependent noise at the sea states shown and above median levels of underwater noise off a beach. Non-motorised recreational water activities are hence likely detectable over some range in aquatic environments. How far exactly these sounds will be detectable, will depend upon the specific environment, its sound propagation characteristics, the spectrum level of ambient noise, and the detection capabilities of the receiver including its receiving directiv- ity index, signal-to-noise threshold, and receiver operating characteristics (see, e.g. [11] for signal detection in noise by hydrophone systems, or [12] for a recent review of signal detection in noise by marine mammals). Spectrum levels are high enough to be detectable by several species of marine megafauna [12].

The recordings in this study were sampled at $32 \mathrm{kHz}$, limiting all spectra to below $16 \mathrm{kHz}$. Previous studies on the sounds emitted by scuba divers during inhalation showed them as broadband up to $125 \mathrm{kHz}$, peaking at $35-80 \mathrm{kHz}$ [13], varying with equipment and diver motion and experience [14]. A closed-circuit underwater breathing apparatus also exhibited broadband energy, peaking at about $10 \mathrm{kHz}$ [15].

The variability of the spectra recorded over all passes and people performing the same activity was about $30-40 \mathrm{~dB}$ (difference between the 5th and 95th PSD percentiles). Sound levels might differ with speed, effort and skill. Listening to the hydrophones in real time while the swimmers were performing their activities, it only took a couple of passes to recognise (by ear only) which activity was happening, and in some cases, who was performing it. It would certainly be interesting to find out whether the noisy swimmers are the faster ones, or whether some of the quieter ones are more efficient. Sanders [16] analysed underwater videos of swimmers of varying skill levels and tracked the undulations of specific points on the body (hip, knee and ankle). "Skilled performance $[\ldots]$ is characterised by sequencing of joint actions to produce a single sinusoidal body wave moving caudally with not decreasing and preferably increasing velocity, low biological noise, and small variability" [16]. Biological noise in this context was determined by Fourier transform of the undulations of the joints and quantified as the power at frequencies other than the fundamental frequency of motion. Our study suggests that underwater bioacoustic noise could also be a measure useful in the assessment of swimming efficiency.

\section{Conclusion}

This article presented underwater sound signatures of recreational water activities, showing that different styles of swimming exhibit differences in spectro-temporal characteristics, by which the styles, and often the individual swimmers, can be told apart. Applications range from swimmer detection to swimmer performance assessment.

Acknowledgments Thank you to Rebecca Wellard, Leila Fouda, Sylvia Osterrieder, Sven Gastauer, and Nicholas Riddoch, all of the Centre for Marine Science \& Technology, for swimming in a cold public pool during the off-season. David Minchin and Malcolm Perry kindly assisted with equipment preparation, calibration, and data collection. The authors are also grateful to Scotch College and the University of 
Western Australia for the use of their pools during tests, and to Mullaloo Surf Lifesavers through BeachLAB and Ocean Reef SeaRescue for measurements conducted off Western Australia's beaches.

Funding This study was funded by the Western Australian Government, Office of Science, under the Applied Research Program-Round 2. The Western Australian Government does not endorse any information, product, process or outcome, arising from or in relation to this study.

\section{Compliance with Ethical Standards}

Conflict of interest The authors declare that they have no conflict of interest.

\section{References}

1. Richardson, W.J., Greene, C.R., Malme, C.I., Thomson, D.H.: Marine Mammals and Noise. Academic Press, San Diego (1995)

2. Wyatt, R.: Review of existing data on underwater sounds produced by the oil and gas industry. Joint Industry Programme on Sound and Marine Life, Seiche Measurements Ltd, Great Torrington, UK (2008)

3. Press, W.H., Teukolsky, S.A., Vetterling, W.T., Flannery, B.P.: Numerical Recipes: The Art of Scientific Computing, 3rd edn. Cambridge University Press, Cambridge, New York (2007)

4. Parsons, M.J.G., Longbottom, S., Lewis, P., McCauley, R.D., Fairclough, D.V.: Sound production by the West Australian dhufish (Glaucosoma hebraicum). J. Acoust. Soc. Am. 134(4), 2701-2709 (2013). doi:10.1121/1.4818775

5. Erbe, C.: Underwater noise of small personal watercraft (jet skis). J. Acoust. Soc. Am. 133(4), EL326-EL330 (2013). doi:10.1121/ 1.4795220
6. Wenz, G.M.: Acoustic ambient noise in the ocean: spectra and sources. J. Acoust. Soc. Am. 34(12), 1936-1956 (1962). doi:10. $1121 / 1.1909155$

7. Longuet-Higgins, M.S.: Bubble noise spectra. J. Acoust. Soc. Am. 87(2), 652-661 (1990). doi:10.1121/1.398934

8. Ross, D.: Mechanics of Underwater Noise. Pergamon Press, New York (1976)

9. Prosperetti, A., Lu, N.Q., Kim, H.S.: Active and passive acoustic behavior of bubble clouds at the oceans surface. J. Acoust. Soc. Am. 93(6), 3117-3127 (1993). doi:10.1121/1.405696

10. Prosperetti, A., Lu, N.Q., Lezzi, A.: Bubble clouds as sources and scatterers of underwater sound. J. Acoust. Soc. Am. 85(S1), S154 (1989). doi:10.1121/1.2026825

11. Urick, R.J.: Principles of Underwater Sound, 3rd edn. McGraw Hill, New York (1983)

12. Erbe, C., Reichmuth, C., Cunningham, K.C., Lucke, K., Dooling, R.J.: Communication masking in marine mammals: a review and research strategy. Mar. Pollut. Bull. 103, 15-38 (2016). doi:10. 1016/j.marpolbul.2015.12.007

13. Lo, K.W., Ferguson, B.G.: Diver detection and localization using passive sonar. In: Proceedings of Acoustics, Fremantle, Western Australia (2012)

14. Donskoy, D.M., Sedunov, N.A., Sedunov, A.N., Tsionskiy, M.A.: Variability of SCUBA diver's acoustic emission. In: Proceedings of SPIE, p. 620408. Orlando, FL (2008)

15. Fillinger, L., Hunter, A.J., Zampolli, M., Clarijs, M.C.: Passive acoustic detection of closed-circuit underwater breathing apparatus in an operational port environment. J. Acoust. Soc. Am. 132(4), EL310-EL316 (2012). doi:10.1121/1.4751985

16. Sanders, R.H.: Kinematics, coordination, variability, and biological noise in the prone flutter kick at different levels of a "learn-toswim" programme. J. Sports Sci. 25(2), 213-227 (2007). doi:10. 1080/02640410600631025 